\title{
An Ultracold Strongly Coupled Gas: a Near-ideal Liquid
}

\author{
Boris A. Gelman, Edward V. Shuryak and Ismail Zahed \\ Department of Physics and Astronomy \\ State University of New York, Stony Brook, NY 11794-3800
}

\begin{abstract}
Feshbach resonances of trapped ultracold alkali atoms allow to vary the atomic scattering length $a$. At very large values of $a$ the system enters an universal strongly coupled regime in which its properties - the ground state energy, pressure etc.-become independent of $a$. We discuss transport properties of such systems. In particular, the universality arguments imply that the shear viscosity of ultracold Fermi atoms at the Feschbach resonance is proportional to the particle number density $n$, and the Plank constant $\hbar: \eta=\hbar n \alpha_{\eta}$, where $\alpha_{\eta}$ is a universal constant. Using Heisenberg uncertainty principle and Einstein's relation between diffusion and viscosity we argue that the viscosity has the lower bound given by $\alpha_{\eta} \leq(6 \pi)^{-1}$. We relate the damping of low-frequency density oscillations of ultracold optically trapped ${ }^{6} \mathrm{Li}$ atoms to viscosity and find that the value of the coefficient $\alpha_{\eta}$ is about 0.3 . We also show that such a small viscosity can not be explained by kinetic theory based on binary scattering. We conclude that the system of ultracold atoms near the Feshbach resonance is a near-ideal liquid.
\end{abstract}

\section{INTRODUCTION}

A presence of the Feshbach resonance in binary collisions of alkali atoms at ultracold temperatures provides an experimental control of an effective strength of the inter-particle interaction. In the case of ultracold dilute atomic systems the effective interaction strength is given by an $s$-wave scattering length $a$. For a gas a "diluteness parameter" $n a^{3}$, where $n$ is the number density, is much less then unity.* An atomic system can be tuned to a regime of the Feshbach resonance using an external magnetic field. On resonance the scattering length and "diluteness parameter" formally diverge. The system can no longer be described as a dilute weakly interacting Fermi or Bose gas. This regime is often referred to as strongly coupled regime.

As external magnetic field is tuned to a critical value corresponding to the Feshbach resonance the effective dimensionless coupling constant describing two-body interaction formally diverges. In the case of ultracold alkali atoms this coupling constant $g$ is given in terms of the $s$ wave scattering length $a$ by $g=4 \pi \hbar^{2} a / m$, where $m$ is the mass of an atom. Since the coupling constant $g$ goes to infinity at the Feshbach resonance it is natural to expect that the thermodynamic properties of the atomic system become independent of $g$ and consequently of the nature of the two-body interaction [1]. Instead, these properties are given in terms of the universal constants times the appropriate dimensional factors which for ultracold atoms are given in terms of the remaining parameters, namely, the Plank constant $\hbar$, particle density $n$, and an atomic mass $m$.

For example, the pressure $p(n)$ of ultracold atoms at the Feshbach resonance is proportional to that of the ideal Fermi gas, $p_{0}(n) \sim n^{5 / 3} / m$,

\footnotetext{
${ }^{*}$ A typical density of ultracold trapped atomic gases is $n \sim 10^{13}-10^{15} \mathrm{~cm}^{-3}$.
}

$$
\frac{p(n)}{p_{0}(n)}=1-\beta
$$

where $\beta$ is an universal dimensionless constant. For ultracold fermionic atoms $\beta \approx 0.5$. The "universality" principle means that the value of $\beta$ is the same for any strongly coupled system of fermions, such as cold atoms or dilute neutron matter. This constant is expected to depend on the particle spin.

An atomic system near the Feshbach resonance is an example of a quantum many-body system with a large or formally infinite dimensionless parameter describing twobody interaction. Other examples include: a strongly coupled quark-gluon plasma (sQGP) [5], a strongly coupled classical electromagnetic plasma and a strongly coupled $\mathcal{N}=4$ supersymmetric Yang-Mills gauge theory. These systems are briefly discussed in section V.

In all of the above systems one finds that the difference between weak and strong coupling regimes in the total (free) energy, although nonzero, is nevertheless much less dramatic than the corresponding difference in their transport parameters-viscosity, diffusion, etc. - which change by orders of magnitude. In these cases the strongly coupled matter behaves as a liquid, with the the mean free path comparable or even smaller than the inter-particle distance.

The goal of this paper is to show that the transport properties of a system of ultracold atoms change dramatically as the system is driven from a weakly interacting regime to the regime of the Feshbach resonance. As we will discuss in details this change is manifested in a steep decrease by about two orders of magnitude of the damping rate of the collective oscillations of a cloud of ${ }^{6} \mathrm{Li}$ atoms near the Feshbach resonance observed in the experiments of Kinast et al. and Bartenstein et al.. This decrease cannot be attributed to the phase transition that the system is expected to undergo at a low enough temperature. The maximum of the oscillations is near the surface of the atomic cloud where the temperature is above the local phase transition temperature. Furthermore, the transport coefficients such as viscosity 
and diffusion display universal properties near the Feshbach similar to the pressure and energy density.

\section{THE SHEAR VISCOSITY OF ULTRACOLD FERMIONIC ATOMS FROM OSCILLATION DAMPING}

In this section we use the equations of hydrodynamics to describe the damping rate of the collective excitations of the ultracold Fermi atoms near the Feshbach resonance. A spectacular manifestation of the hydrodynamical behavior of ultracold optically trapped atomic clouds near the Feshbach resonance is the elliptic flow observed after the trap is switched off $[6,7]$. Such flow is also observed at higher temperatures. Thus it is not by itself related to quantum low-temperature phenomena like condensation or superfluidity but rather it is a manifestation of a strongly coupled regime. A good quantitative description of the elliptic flow was obtained in a framework of ideal hydrodynamics (i.e. neglecting viscosity). In principle, this comparison by itself may provide an upper limit on the viscosity.

Additional evidence for the hydrodynamical behavior is provided by the experiments of Kinast et al. [2,3] and Bartenstein et al. [4] on collective oscillation of ultracold trapped ${ }^{6} \mathrm{Li}$ atoms in the vicinity of the Feshbach resonance. The measured frequencies of the lowest modes are in a good agreement with the predictions of ideal non-suprefluid hydrodynamics.

Our main point is that the damping of these collective excitations can be described by including the dissipative (in particular, viscous) terms in the hydrodynamic equations. As will be shown in the next section, the viscous terms are very small at least for the low frequency collective excitations. Thus, these terms can be included perturbatively.

If the collision rate of atoms is large enough to establish a local equilibrium the collective vibrations of the atomic cloud can be described using standard hydrodynamical theory [9]. The collective vibrations are described by the local density $n(\vec{r})$, pressure $p(\vec{r})$ and velocity $\vec{v}(\vec{r})$ which are the solutions of the continuity equation, Euler equations of motions and the equation of state, respectively,

$$
\begin{aligned}
& m \frac{\partial n}{\partial t}+\nabla \cdot(m n \vec{v})=0, \\
& m n \frac{\partial \vec{v}}{\partial t}+m n(\vec{v} \cdot \nabla) \vec{v}=-\nabla p-n \nabla V, \\
& p=A n^{\gamma+1},
\end{aligned}
$$

where $V=(1 / 2) m \sum_{i} \omega_{i}^{2} r_{i}^{2}$ is the harmonic trapping potential, $A$ is a constant, and $\gamma$ is the polytropic index. As discussed in [1] a cold gas of strongly interacting fermions at the has a universal equation with polytropic index $\gamma=2 / 3$.

The lowest collective modes correspond to the small vibrations of the density, pressure and velocity around their equilibrium values, $n_{e q}, p_{e q}$ and $\vec{v}_{e q}=0$. These values are determined by the static limit of the Eqs. (2) in which the Euler equation takes the form $\nabla p_{e q}+n_{e q} \nabla V=$ 0 . The equilibrium density is

$$
n_{e q}(r)=n_{e q}(0)\left(1-\sum_{i=1}^{3} \frac{r_{i}^{2}}{R_{i}^{2}}\right)^{1 / \gamma}
$$

for $\vec{r}$ inside the ellipsoid

$$
\frac{x^{2}}{R_{x}^{2}}+\frac{y^{2}}{R_{y}^{2}}+\frac{z^{2}}{R_{z}^{2}}=1
$$

and zero outside. In equation $(3) n_{e q}(0)$ is the equilibrium density of the atomic cloud at the center of the harmonic trap $(\vec{r}=0)$ and

$$
R_{i}=\sqrt{\frac{p_{e q}(0)}{n_{e q}(0)} \frac{2(\gamma+1)}{\gamma m \omega_{i}^{2}}}
$$

are the radii of the cloud with $p_{e q}(0)$ being the equilibrium central value of the pressure. These radii can be expressed in terms of the chemical potential $\mu$. Using the Gibbs-Duham relation, $d p=n d \mu$ (valid at constant temperature) and the equation of state, $p=A n^{\gamma+1}$, one can easily show that

$$
\frac{p}{n}=\frac{\gamma}{\gamma+1} \mu
$$

so that the Thomas-Fermi radii are

$$
R_{i}=\sqrt{\frac{2 \mu}{m \omega_{i}^{2}}}
$$

independent of $\gamma$.

The chemical potential $\mu$ in Eqs. (6) and (7) includes interactions of the trapped atoms in the vicinity of the Feshbach resonance. The effect of the interaction in the unitary limit is to scale the chemical potential of the noninteracting trapped Fermi gas $[6,8]$ :

$$
\mu=\mu^{(0)} \sqrt{1-\beta}
$$

where $\beta \approx 0.5$ is the universal constant discussed in the introduction and $\mu^{(0)}$ is the chemical potential of $N$ spin$1 / 2$ noninteracting fermions confined in a harmonic trap $[10]$

$$
\mu^{(0)}=\hbar \bar{\omega}(3 N)^{1 / 3}=k_{B} T_{F}
$$

where $\bar{\omega}^{3}=\omega_{1} \omega_{2} \omega_{3}$ and $k_{B}$ is the Boltzmann constant. The last equation also defines the Fermi temperature $T_{F}$ of the system.

Linearizing Eqs. (2) to describe small density $n=$ $n_{e q}+\delta n e^{i \omega t}$ and velocity $\vec{v} e^{i \omega t}$ oscillations one can obtain the following equations for the density and velocity amplitudes: 


$$
\begin{aligned}
-m \omega^{2} \delta n & =\nabla \cdot\left(n_{e q} \nabla\left(\frac{1}{n_{e q}} \frac{d p}{d n} \delta n\right)\right) \\
-\omega^{2} \vec{v} & =\nabla(\vec{v} \cdot \nabla V)+\gamma \nabla \cdot \vec{v} \nabla V .
\end{aligned}
$$

The form of the collective vibrational modes which are the solutions of Eq. (10) depend on the symmetry of the trap potential. If the confining potential is isotropic, $\omega_{1}=\omega_{2}=\omega_{3}$, the collective modes have spherical symmetry and can be characterized by definite angular momentum and its $z$ component, $l$ and $m$. The monopole mode, $l=m=0$, has a velocity profile which is proportional to $\vec{r}$. Such modes are referred to as breathing modes. The dipole mode $l=1$ involves the motion of the center of mass of the cloud and is not usually excited in the experiments we are interested in. There are five degenerate quadrupole modes corresponding to $l=2$ with $m=0, \pm 1, \pm 2$.

We will discuss the collective modes that are excited in an axially symmetric trap with $\omega_{1}=\omega_{2}=\omega_{r}$ and $\omega_{3}=\omega_{z}=\lambda \omega_{r}$, where $\lambda$ is a constant. In the experiment of Bartenstein et al. [4] $\lambda$ is $0.03^{\dagger}$. In such traps the collective modes corresponding to different angular momenta but the same $z$-component $m$ are mixed.

To find these modes we look for the solutions of Eq. (10) in the form

$$
\vec{v}=\left(a_{x} x, a_{y} y, a_{z} z\right)=\left(a_{r} x, a_{r} y, a_{z} z\right) .
$$

The set of equations in Eq. (10) for $\vec{v}$ reduces to the secular equation for the eigenfrequencies and the corresponding eigenvectors. The three frequencies are [11],

$$
\begin{aligned}
\omega_{1,2}^{2} & =\omega_{r}^{2}\left(1+\gamma+\frac{1}{2}(\gamma+1) \lambda^{2}\right. \\
& \left. \pm \frac{1}{2} \sqrt{(\gamma+2)^{2} \lambda^{4}+\left(\gamma^{2}-3 \gamma-2\right) \lambda^{2}+4(\gamma+1)^{2}}\right) \\
\omega_{3} & =\sqrt{2} \omega_{r},
\end{aligned}
$$

where in the first equation the plus and minus signs correspond to axial and radial modes with frequencies $\Omega_{z}=\omega_{1}$ and $\Omega_{r}=\omega_{2}$ respectively. The third frequency (which is the same as one of the frequencies in the case of the spherical trap) is the frequency of the two remaining degenerate modes with $l=2, m= \pm 1 \pm 2$. For the cigar-shaped traps, i.e. in the limit of a very small $\lambda$, the axial and radial frequencies reduce to

$$
\begin{aligned}
& \frac{\Omega_{z}}{\omega_{z}}=\sqrt{3-\frac{1}{\gamma+1}}, \\
& \frac{\Omega_{r}}{\omega_{r}}=\sqrt{2 \gamma+2} .
\end{aligned}
$$

\footnotetext{
${ }^{\dagger}$ Such traps with $\lambda \ll 1$ are referred to as cigar-shaped or prolate.

${ }^{\ddagger}$ Such flow with velocity components proportional to the position is often referred to as Hubble flow.
}

For the equation of state with the polytropic index $\gamma=$ $2 / 3$. the axial and radial frequencies are, respectively,

$$
\begin{aligned}
& \frac{\Omega_{z}}{\omega_{z}}=\sqrt{\frac{12}{5}}=1.549, \\
& \frac{\Omega_{r}}{\omega_{r}}=\sqrt{\frac{10}{3}}=1.826 .
\end{aligned}
$$

The corresponding velocities, Eq. (11), in the limit as $\lambda \rightarrow 0$ are given for the axial mode by,

$$
\frac{a_{z}}{a_{r}}=-\frac{2(1+\gamma)}{\gamma}=-5
$$

and for the radial mode by,

$$
\frac{a_{z}}{a_{r}}=\frac{\gamma}{\gamma+1} \lambda^{2}=\frac{2}{5} \lambda^{2}
$$

where $\gamma=2 / 3$ was used.

The frequency of the axial mode in Eq. (14), agrees very well with the value of approximately 1.55 measured by Bartenstein et al. [4] The frequency of the radial mode at the Feshbach resonance measured by Kinast et al. $\left(\Omega_{r} / \omega_{r}=1.829\right)$ also agrees remarkably well with the value given in Eq. (14) [3].

However, the frequency of the radial mode measured in the experiment by Bartenstein et al. ( [4]) is 1.67 which is about $20 \%$ below the corresponding value in Eq. (14). This deviation complements a sudden jump in the frequency and especially in the damping rate of the radial mode [4] not far from the resonance on the "BCS" side. There is no jump at this particular value of the scattering length in the axial mode, so this phenomenon cannot possibly be associated with a change in the equation of state or transport properties by itself. Thus, an extra source of dissipation in the radial mode in the experiment by Bartenstein et al. must thus be associated with a non hydrodynamical effects . $^{\S}$

We now apply viscous hydrodynamics to describe the damping of the collective modes in the vicinity of the Feshbach resonance. The primary source of dissipation in the hydrodynamic limit is shear viscous flow [12]. The rate of change of the total energy of a mode is given by [9]

$$
\begin{aligned}
\left\langle\frac{d E}{d t}\right\rangle & =-\int \frac{\eta}{2}\left(\frac{\partial v_{i}}{\partial x_{k}}+\frac{\partial v_{k}}{\partial x_{i}}-\frac{2}{3} \delta_{i k} \nabla \cdot \vec{v}\right)^{2} d^{3} r \\
& =-\frac{2}{3} a_{r}^{2}\left(1-\frac{a_{z}}{a_{r}}\right)^{2} \int \eta(r) d^{3} r
\end{aligned}
$$

where $\eta$ is the coefficient of the shear viscosity and the ratio $a_{z} / a_{r}$ is given in Eqs. (16) for each mode.

\footnotetext{
${ }^{\S}$ We are grateful to R. Grimm for a discussion about a possible source of this effect.
} 
A damping rate of a collective mode can now be obtained by dividing the rate of change of energy, Eq. (17) by half of the the time-averaged total energy of the vibrational mode which is equal to maximum kinetic energy of the mode

$$
\begin{aligned}
\langle E\rangle & =\frac{m}{2} \int n_{e q}(\vec{r}) v^{2}(\vec{r}) d^{3} r \\
& =\frac{\pi^{2}}{128} m n_{e q}(0) a_{r}^{2} R_{x} R_{y} R_{z}\left(R_{x}^{2}+R_{y}^{2}+\frac{a_{z}^{2}}{a_{r}^{2}} R_{z}^{2}\right) \\
& =\frac{1}{16} m N a_{r}^{2}\left(2 R_{r}^{2}+\frac{a_{z}^{2}}{a_{r}^{2}} R_{z}^{2}\right)
\end{aligned}
$$

where Eq. (3) was used with $\gamma=2 / 3$. In the last step the product of Thomas-Fermi radii was expressed in terms of the total number of particles $N$ in the cloud,

$$
\begin{aligned}
N & =\int n_{e q}(\vec{r}) d^{3} r=\int n_{e q}(0)\left(1-\sum_{i=1}^{3} \frac{r_{i}^{2}}{R_{i}^{2}}\right)^{3 / 2} d^{3} r \\
& =\frac{\pi^{2}}{8} n_{e q}(0) R_{x} R_{y} R_{z}
\end{aligned}
$$

and $R_{x}=R_{y}=R_{r}$ for the axially symmetric trap.

Using Eqs. (17) and (18) the damping rate is

$$
\begin{aligned}
\Gamma & =\frac{1}{2}\left|\frac{\langle d E / d t\rangle}{\langle E\rangle}\right| \\
& =\frac{16}{3 m N} \frac{\left(1-\frac{a_{z}}{a_{r}}\right)^{2}}{\left(2 R_{r}^{2}+\frac{a_{z}^{2}}{a_{r}^{2}} R_{z}^{2}\right)} \int \eta(r) d^{3} r .
\end{aligned}
$$

This expression will be used in the next section to extract the coefficient of shear viscosity of the strongly coupled atoms near the Feshbach resonance.

\section{QUANTUM VISCOSITY}

\section{A. Generalities}

In the weak coupling regime of a small scattering length $a$, transport properties are determined by the particle mean-free path

$$
l_{\mathrm{mfp}}=\frac{1}{n \sigma}, \quad \sigma=4 \pi a^{2} .
$$

In the strong coupling regime, when $a \rightarrow \pm \infty$, Eq. (21) becomes meaningless. Since the cross section does not diverge but is bounded by unitarity, this regime is sometimes referred to as "unitarity limited" one. Indeed, the maximal possible two-body cross section is limited by $\sigma_{\max }=4 \pi / k^{2}$ for fixed collision energy ( $k$ is the wavenumber of relative motion). Thus, one may think that the mean free path is actually $l_{\min }=1 / n \sigma_{\max }$. However, this is too naive as well, since at strong coupling there is no reason to limit kinetics to a picture of propagating particles rarely suffering only binary collisions.

Whatever the microscopic structure of matter may be, the viscous hydrodynamics is an adequate description of low frequency dynamics. It is important that it is based on expansion in inverse powers of the cross sections, or expansion in small mean free paths $l$, so the stronger the interaction the better this approach is expected to work. The viscosity is in general defined via the dissipative part of the stress tensor and can be defined without any assumption on the underlying matter. It appears in observables like the sound dispersion law

$$
\omega=c_{s} k+\frac{i}{2} \frac{4 \eta}{3 m n} k^{2}
$$

and thus can be measured. In fact, the damping rate in eq. (20) is the corresponding analog for trapped atoms.

\section{B. Universality of transport coefficients}

For simplicity we discuss mostly the zero temperature limit in this section. The temperatures in the experiments of of Kinast et al. [2,3] and Bartenstein et al. [4] range from $0.1 T_{F}$ to $0.03 T_{F}$.

One of the basics of low temperature physics of weakly coupled systems is that at low $T$ the mean free path of quasiparticles goes to infinity, together with viscosity. There are multiple examples of such behavior, e.g. in liquid ${ }^{4} \mathrm{He}$ the minimal viscosity is around $T_{c}$ and then it rises as $T \rightarrow 0$. The normal component at low $T$ is thus very viscous.

However, this picture is no longer true at weak coupling. Strongly coupled normal component is also expected to have low viscosity even at low $T^{* *}$. Furthermore, we suggest that such oscillations even at $T \rightarrow 0$ would have a damping described by a "quantum viscosity" proportional to the Plank constant, reflecting limitation on particle localization even at zero temperature. Indeed, at zero temperature a single interaction parameter - the scattering length $a$ - diverges at the Feshbach resonance. The only remaining length scale is given by the inter-particle distance, $n^{-1 / 3}$. Thus, the mean free path must be of this scale.

These arguments lead to universal relations for transport coefficients. In particular, the only form of the viscosity is

$$
\eta=n \hbar \alpha_{\eta}
$$

\footnotetext{
${ }^{* *}$ For clarity, we remind again that experimentally observed oscillations are not an elementary quantum phonon state, but a highly excited state to which we apply the viscous hydrodynamics.
} 
where an universal dimensionless coefficient $\alpha_{\eta}$ is introduced. Note that it is independent on the particle mass. The same arguments are also valid for strongly coupled bosonic atoms, although of course with a different $\alpha_{\eta}$.

At nonzero temperature viscosity has a similar form,

$$
\eta=n \hbar \alpha_{\eta}+s(T) \hbar \beta_{\eta}
$$

where the second temperature-dependent term contains the entropy density and another universal coefficient $\beta_{\eta}$. This second term reproduces the finite temperature. dependence of the damping observed by the Duke group, although we do not have any other justification for this form of this term. We repeat, that none of these terms are reproduced by binary collisions, neither in magnitude nor in parametric dependences.

Similarly, the characteristic time scale in strongly coupled liquid is given by the Fermi energy and the Plank constant $\hbar, \tau^{-1} \sim \epsilon_{F} / \hbar \sim \hbar n^{2 / 3} / m$. even if it include purely Bosonic atoms. Thus, the scattering rate should be simply proportional to this scale with another universal coefficients, although different for bosons and fermions.

The next question is what are the values of these dimensionless parameters. For an ordinary liquid, these constants are of order one. For a quantum liquid, such as ${ }^{4} \mathrm{He}$ with a large quasiparticle mean free path the value of $\alpha_{\eta}$ exceeds that of ordinary liquids by about three orders of magnitude.

If the dimensionless parameters are much smaller than one, we deal with exotic near-ideal liquid. Although the available data available is not conclusive. It is is quite possible that the ultracold fermionic atoms near the Feshbach resonance are in the regime of near-ideal liquid.

\section{Experimental estimate for universal viscosity coefficient}

It was shown in section II that the damping rate of the small collective oscillations is proportional to the volumeintegrated viscosity, Eq. (20). The universality relation Eq. (23) reduces the integral in Eq. (20) to the total number of particles times the coefficient $\alpha_{\eta}$ which we want to determine. Thus, the damping rate is ${ }^{\dagger \dagger}$,

$$
\Gamma=\frac{16}{3 m} \frac{\left(1-\frac{a_{z}}{a_{r}}\right)^{2} \hbar \alpha_{\eta}}{\left(2 \lambda^{2}+\frac{a_{z}^{2}}{a_{r}^{2}} R_{z}^{2}\right)} .
$$

\footnotetext{
${ }^{\dagger \dagger}$ The universal relation should not be valid near the edges of the system, at less than one mean-free-path or at optical depth less than one, where dissipation is larger. However, the edge includes only about one percent of particles and their contribution can be neglected.
}

For the axial mode in the cigar-shaped potential trap using the ratio $a_{z} / a_{r}$ from Eq. (15), the coefficient $\alpha_{\eta}$ is

$$
\alpha_{\eta} \approx \frac{25 m R_{z}^{2} \Gamma_{z}}{192 \hbar}
$$

The experimental values are $\Gamma_{z} / \omega_{z}=0.0036$ at the point of the Feshbach resonance, with the minimal value of $\left.\left(\Gamma_{z} / \omega_{z}\right)\right|_{\min }=0.0015$ slightly off the resonance [4]. Using the axial trap frequency $\omega_{z} \approx 140 \mathrm{~Hz}$ we get our final result for the minimal dimensionless viscosity $\ddagger$

$$
\left.\alpha_{\eta}^{(z)}\right|_{\min } \approx 0.3 .
$$

The fact that we get a number of order unity is an indication that the suggested "quantum viscosity" indeed exists and is described by the universality arguments.

For the radial mode, using Eq. (16) and the fact that $R_{z}=R_{r} / \lambda$ we get,

$$
\alpha_{\eta} \approx \frac{3 m R_{x}^{2} \Gamma_{r}}{8 \hbar} .
$$

In the experiment of Bartenstein et al. [4] with the radial trap frequency $\omega_{r} \approx 4700 \mathrm{~Hz}$ the damping rate of the radial mode at the Feshbach resonance is $\Gamma_{r}=$ $0.0625 \omega_{r}$. Thus, the coefficient $\alpha_{\eta}$ extracted from the damping rate of the radial is

$$
\alpha_{\eta}^{(r)} \approx 1.1
$$

The coefficient $\alpha_{\eta}$ extracted from the damping rate of the radial mode measured in the experiment of Kinast et al. $[3]$ is

$$
\alpha_{\eta}^{(r)} \approx 0.2
$$

The damping rate of the radial mode measured by Kinast et al. [3] at temperature comparable to the Fermi energy is more or less consistent with an estimate from kinetic theory discussed in the next section.

Kinast et al. [3] measured the temperature dependence of the damping rate. As the temperature is lowered the damping rate decreases in contradiction to the kinetic estimates in section IV, but in agreement with our reasoning. Unfortunately, the temperature is not small enough to conclude whether the universal "quantum viscosity" regime at $T=0$ is reached and $\alpha_{\eta} \neq 0$ or not, and whether the same universal viscosity is responsible for damping of both axial and radial modes. The second term in Eq. (24) proportional to the entropy density can explain this data if value of $\beta_{\eta}$ is of order unity.

\footnotetext{
${ }^{\ddagger \ddagger}$ The error is comparable to the value itself, as can be seen from experimental data points. Ironically, the situation with dimensionless viscosity of quark-gluon plasma is quite similar.
} 


\section{COMPARISON WITH THE TRADITIONAL KINETIC THEORY}

In this section we will show that the kinetic theory based on the notion of binary collisions fails to describe the damping of the axial mode discussed above.

Let us start with an order of magnitude estimates of the collision rates and viscosity neglecting Pauli blocking and using the largest "unitary limited" cross section $\sigma=4 \pi / k_{F}^{2}$. The collision rate at the center of the trap estimated like this gives

$$
\tau_{\text {coll }}^{-1}=n(0) \sigma v_{f} \sim 10^{5} s^{-1}
$$

where the last number corresponds to conditions of the experiment of Bartenstein et al. [4]. Compared with oscillation frequencies, $\omega_{r}=4712 \mathrm{~Hz}, \omega_{z}=141.9 \mathrm{~Hz}$ of the trap, which leads to a conclusion that only the latter mode have a chance to be hydrodynamical.

The mean free path, $l_{\mathrm{mfp}}$, of a particle is of order $1 /(n \sigma)$, while the shear viscosity is

$$
\eta \sim m \bar{v} n l_{\mathrm{mfp}}=\frac{m v}{\sigma}
$$

where $v$ is the average velocity of a particle. In the limit of zero temperature the velocity is set by the Fermi momentum, $m v_{F}=\hbar k_{F}$. In the vicinity of the Feshbach resonance the cross section is unitary bounded, $\sigma<\sigma_{\max }=4 \pi / k_{F}^{2}$. So, if we take its maximal value (and still ignore for a moment Pauli blocking), we will get a minimal viscosity which may follow from binary collisions:

$$
\frac{\eta}{\hbar n}>\frac{40}{6 \pi}
$$

This inequality is strongly violated in experiment, as shown above: this "minimal binary" value is in fact larger than observed value, and forty times larger than the derived bound possible for a liquid.

Furthermore, if the fermionic atoms were in the Fermi liquid regime, the collision rate will be significantly lowered by Pauli blocking which should lead to a suppression factor about $\left(T / T_{F}\right)^{2} \sim 1 / 1000$ in the experimental conditions. If true, the oscillations then would be basically collisionless and no hydro phenomena would be present. In a picture of BCS-type pairing, with relatively small modification of Fermi sphere, $T$ in the above formula to be substituted by a gap, so the rescattering suppression would be of the order of $\left(\Delta / \epsilon_{F}\right)^{2}$. The gap value is not well known, but this suppression factor is still about $1 / 100$ or so. We must then conclude that both pictures are wrong and in fact there seem to be no Pauli blocking whatsoever ${ }^{\S}$.

\footnotetext{
$\S^{\S}$ After observation of elliptic flow this issue was discussed
}

To make more quantitative conclusion we will derive here the damping rate of a collective mode in a axially symmetric trap applying the traditional kinetic equation to an almost ideal Fermi gas with unitary limited cross section.

A damping rate of a collective mode in the kinetic theory is determined by a relaxation time which is a measure of how fast a particle distribution function $n(\vec{p}, \vec{r}, t)$ for a given collective mode takes an equilibrium form. Both the time dependent and equilibrium distribution functions are the solutions of the kinetic equation. The equilibrium distribution for a Fermi gas is

$$
n(\epsilon, \vec{r})=\left(e^{(\epsilon-\mu+V(r)) / k_{B} T}+1\right)^{-1},
$$

where $\epsilon=p^{2} / 2 m$.

During an oscillation the distribution function is different from the equilibrium one. The collisions between particles cause the non-equilibrium distribution function to "relax" to the equilibrium form. These collisions are the source of the damping of the oscillations.

As shown in [10] the damping rate of the oscillations of Fermi gases is equal to

$$
\Gamma=\frac{\left\langle\left(p_{1, z}^{2}-\frac{p_{1}^{2}}{3}\right) \Gamma\left[p_{1, z}^{2}-\frac{p_{1}^{2}}{3}\right]\right\rangle}{\left\langle p_{1, z}^{2}-\frac{p_{1}^{2}}{3}\right\rangle},
$$

where

$$
\begin{aligned}
& \left\langle\left(p_{1, z}^{2}-\frac{p_{1}^{2}}{3}\right) \Gamma\left[p_{1, z}^{2}-\frac{p_{1}^{2}}{3}\right]\right\rangle= \\
& \frac{1}{4(2 \pi \hbar)^{6}} \int d^{3} r d^{3} p_{1} d^{3} p_{2} d^{3} p_{1}^{\prime} d^{3} p_{2}^{\prime}(\Delta \Phi)^{2} \\
& W \delta^{3}\left(\vec{p}_{1}+\vec{p}_{2}-\vec{p}_{1}^{\prime}-\vec{p}_{2}^{\prime}\right) \delta\left(\epsilon_{1}+\epsilon_{2}-\epsilon_{1}^{\prime}-\epsilon_{2}^{\prime}\right) \\
& n_{1} n_{2}\left(1-n_{1}^{\prime}\right)\left(1-n_{2}^{\prime}\right),
\end{aligned}
$$

and

$$
\left\langle\left(p_{z}^{2}-\frac{p^{2}}{3}\right)^{2}\right\rangle=\int d^{3} r d^{3} p(\Phi)^{2} n(1-n),
$$

where $\Delta \Phi=\left(\Phi_{1}+\Phi_{2}\right)-\left(\Phi_{2}^{\prime}+\Phi_{2}^{\prime}\right)$ and the function $\Phi=p_{z}^{2}-\frac{p^{2}}{3}$ describes the deviation of the distribution function of a collective mode from the equilibrium one. The function $W$ is proportional to the scattering amplitude of binary collisions $\vec{p}_{1}, \vec{p}_{2} \rightarrow \vec{p}_{1}^{\prime}, \vec{p}_{2}^{\prime}$. In the vicinity of the Feshbach resonance this function is determined by unitary limit of the scattering amplitude and is equal to

in literature and the MIT group [13] have argued that this might have been due to strong deformation of Fermi sphere in exploding gas. This explanation obviously would not be applicable to small amplitude oscillation we study here. 


$$
W=\frac{\hbar^{2}}{m^{2}} \frac{(2 \pi \hbar)^{3}}{p^{2}}
$$

$\vec{p}=\vec{p}_{1}-\vec{p}_{2}$ is the relative momenta of two particles.

At a very low temperatures the Pauli blocking factors, $n(1-n)$, in Eqs. (36) and (37) significantly reduce the phase space of particles whose collisions appreciably contribute to the relaxation. The main contribution is from the collisions of particles whose momenta lies very close to the Fermi surface:

$$
\epsilon-\mu+V(r) \sim k_{B} T
$$

where $\mu$ is the chemical potential Eq. (9).

After a lengthy but a straightforward calculation one obtains for a damping rate:

$$
\Gamma \approx \frac{9 \pi}{50} \frac{\left(k_{B} T\right)^{2}}{\hbar \mu} .
$$

It is important to note a temperature dependence of the damping rate. It has a typical $T^{2}$ dependence which comes from the life time of weekly interacting Fermi particles or quasi-particles in the case of the Fermi liquid [9]. We stress that such scaling is true only for weekly interacting gas of particles or quasi particles which is observed in liquid ${ }^{3} \mathrm{He}$. If the atomic cloud near Feshbach resonance is indeed a strongly interacting near perfect liquid the damping rate and other dissipative processes will very weekly depend on the temperature. This prediction can be checked experimentally.

The damping rate can be expressed in terms of the trap frequencies and the Fermi temperature $T_{F}$ as

$$
\Gamma \approx \frac{9 \pi}{50}(3 N)^{1 / 3} \bar{\omega}\left(\frac{T}{T_{F}}\right)^{2}
$$

For the experiment of Bartenstein et al. [4] the above formula predicts for the axial mode

$$
\frac{\Gamma_{z}}{\omega_{z}} \approx 0.56
$$

This is much larger than the damping observed close to the Feshbach resonance, and is only compatible with data well away from it, where $k_{F} a<1$.

Similarly, for the experiment of Kinast et al. [3] the Eq. (41) predicts the ratio for the damping of the radial mode to be

$$
\frac{\Gamma_{r}}{\omega_{r}} \approx 0.17,
$$

which is almost ten times larger then the observed ratio $\Gamma_{r} / \omega_{r} \approx 0.014$.

Thus, the strongly interacting atomic cloud is better described by a picture of a nearly perfect strongly interacting liquid with viscosity very close to the minimum possible value. As one moves away from the Feshbach resonance into regime of weekly interacting Fermi gas the kinetic theory becomes again applicable. Furthermore, as detuning gets larger than used in the experiment discussed, the scattering length is getting small, the gas will enter an almost collisionless regime, and the damping rates is getting small again. Thus the damping rate is expected to reach a maximum value for certain value of the magnetic field, separating weakly and strongly coupled regimes.

For the radial mode the value predicted by the same kinetic calculation is

$$
\frac{\Gamma_{r}}{\omega_{r}} \approx 0.02,
$$

comparable to the value of about 0.06 obtained experimentally. It shows that this mode may not be treated by hydrodynamics.

\section{DISCUSSION}

\section{A. Other strongly coupled systems}

In the introduction we mentioned a number of strongly coupled systems which display similar transport properties. One example is the strongly coupled quark-gluon plasma (sQGP) [5] which was found in heavy ion experiments at RHIC at temperatures above the critical temperature, $T=(1-2) T_{c}=170-350 \mathrm{MeV}$. In ultrarelativistic heavy ion collisions also quite spectacular explosions are observed, with radial and elliptic flows surprisingly well described by ideal hydrodynamics. The sQGP seems to have a very small viscosity, i.e. $\eta / s \approx 0.1-0.2$ $[14,15]$ and $\eta / s \approx 0.2-0.4$ from lattice simulations [16]. It is not even far from the lowest limit discussed below. Two of us suggested that the sQGP has such low viscosity because of the existence of weakly bound states near the so called "zero binding lines" on the QCD phase diagram [5]. It was gratifying to learn after that that similar role for trapped atoms is played by Feshbach resonances.

Other examples are: strongly coupled $\mathcal{N}=4$ Supersymmetric Yang-Mills (SYM) gauge theory, a four dimensional conformal field theory (CFT) and the strongly coupled QED plasma. The classical one-component QED plasma is particularly well studied and is known at values of the effective coupling $\Gamma=(Z e)^{2} n^{1 / 3} / T=2-300$ to be a liquid, with a very small viscosity which has its minimum at $\Gamma \sim 10$ (see e.g. [17]).

The CFT is a toy model, emerged mostly in the context of string theories. It is a model resembling QCD, the gauge theory of strong interaction. In CFT as opposed to in QCD the gauge coupling is not "running" with energy scale. Thus, the strong coupling regime is obtained simply by using a large coupling constant in the Lagrangian, $\lambda=g^{2} N$. (Here $g$ is the gauge coupling and $N$ is the number of colors. Only this combination appears if $N$ is large.) This limit can be addressed using the AdS/CFT correspondence as originally suggested by 
Maldacena [18], whereby the quantum intricacies of the strongly coupled gauge theory are mapped onto a classical problem in gravity albeit in ten dimensions. The finite temperature version of this theory describes a plasmalike phase with strongly coupled constituents. The four dimensional world (in which the CFT fields live) is a surface in ten dimensional space, at some distance from a black hole, with a mass adjusted to yield the desired temperature $T$ at this surface. We would like to mention two important results that follow from this construction. One is the equation of state of the underlying gauge theory at strong coupling $\lambda \gg 1$ [19]

$$
\frac{p_{\lambda}(T)}{p_{0}(T)}=\left(1-\frac{1}{4}+\mathcal{O}\left(1 / \lambda^{3 / 2}\right)\right),
$$

where $p_{0}(T) \sim T^{4}$ is the Stephan-Boltzmann pressure for zero coupling. The second result is the viscosity of the underlying gauge theory at strong coupling [20]

$$
\lim _{\lambda \rightarrow \infty} \frac{\eta}{\hbar s}=\frac{1}{4 \pi}\left(1+\mathcal{O}\left(1 / \lambda^{3 / 2}\right)\right)
$$

given in units of the free entropy density $\mathrm{s}^{* * *}$. The corrections were recently calculated in [21].

While the pressure is only changed by $1 / 4$ when the coupling is changed from zero to infinity, the viscosity $\eta / s$ changes from infinity to a finite (and surprisingly small) number. Thus, one may wonder whether other strongly coupled systems show similar behavior, and whether such limiting numbers can be universal and theoretically understood.

The holographic principle in the Maldacena limit and the Kubo formulae show that the viscosity is proportional to the graviton absorption cross section in bulk by the black hole, while (according to Beckeinstein-Hawkins argument) the free entropy is related to its area. As a result the same limit for the viscosity holds for a number of backgrounds, even in different space dimensions. These observations led to a conjecture that Eq. (46) is a universal lower bound valid for any thermal system in strong coupling [22]. Below we provide its heuristic derivation of based on the uncertainty relation and Einstein's famous relation between the diffusion constant from the fluid viscosity. As a result, we show how the bound (46) fits well into the the liquid-like picture of CFT at finite temperature. We then use these insights to derive an even lower bound for cold Fermi systems, and conjecture that at strong coupling they also make an universal near-ideal liquids.

\footnotetext{
${ }^{* * *}$ In thermal gauge theories as in the case of blackbody radiation, there is no ordinary particle density but only the entropy density $s \sim T^{3}$.
}

\section{B. Bounds on Transport Coefficients}

For atomic systems one may also think that as the interaction strength is driven to infinity the transport parameters such as viscosity become as small as possible.

In a weak coupling limit the viscosity and diffusion coefficients are both related to the scattering length and are thought of as proportional to each other. For liquids one should think differently, An example of quite an opposite relation between them was provided by the famous Einstein relation, which we now derive for consistency of the presentation.

The distribution of suspended particles in a thermalized column of gas is given by statistical mechanics. Indeed, if $n(x)$ is the suspension density at finite temperature then

$$
\frac{n(x)}{n(0)}=e^{-m g x / k_{B} T}
$$

which follows from Boltzmann. Einstein's observed that an arbitrary sphere of radius $r_{0}$ in suspension within the column would also follows the same "distribution" profile. The idea is that the sphere under gravity will fall with a terminal Stokes velocity

$$
v_{T}=\frac{m g}{6 \pi r_{0} \eta}
$$

but the fall will be balanced by random upward kicks due to Brownian motion. In equilibrium, the upward diffusion balances the downward gravitational fall so that in the stationary limit

$$
D \frac{d n}{d x}=-n v_{T}
$$

from which it follows that $n(x) / n(0)=e^{-v_{T} x / D}$. Comparing this result with Eq. (47) and using Eq. (48) yields the Einstein's formulae

$$
D=\frac{k_{B} T}{6 \pi r_{0} \eta} .
$$

Although this formulae was derived for a macroscopic sphere of radius $r_{0}$ immersed in a suspension, empirically it is known to hold through 14 orders of magnitude changes down to the suspension constituent wavelength [23].

We recall that in three dimensions the diffusion constant is just $D=v^{2} \tau / 3=\lambda^{2} /(3 \tau)$ where $\lambda$ and $\tau$ are the mean-free path and collision time ${ }^{\dagger \dagger \dagger}$. Inserting this result into Eq. (50) yields

\footnotetext{
${ }^{\dagger \dagger} \mathrm{We}$ note that in $p$ space-dimensions the diffusion constant is $D=\lambda^{2} / p \tau$ and (50) should be derived accordingly. All the bounds to follow can be extended readily to $p$ space dimensions.
} 


$$
\frac{\eta}{1 /\left(r_{0} \lambda^{2}\right)}=\frac{1}{2 \pi}\left(k_{B} T \tau\right) .
$$

In a densely packed liquid the smallest jump (the mean-free path $\lambda$ ) is the size of the quasiparticles $r_{0}$. Classically in densely packed hard balls $\tau$ can be as small as zero due to the fact that they are always touching. Quantum mechanically however this is not allowed since the time localization cannot be better than the limit set by the largest allowed energy, by the Heisenberg uncertainty principle, i.e. $k_{B} T \tau \geq \hbar / 2$. Inserting this result into Eq. (51) yields Eq. (46) since the entropy per unit volume $s$ is just the number of (quasi)particles per unit volume due to the close packing, i.e. $s / k_{B}=n=1 / \lambda^{3}$. The ensuing physical picture of the strongly coupled thermal system is that of a liquid with the shortest time correlation length $\tau_{\text {min }}=\hbar / 2 k_{B} T$.

Our heuristic derivation of Eq. (46) follow from the assumption that Eq. (50) holds for the liquid particles, since the relation is known to hold over many orders of magnitude changes in $\eta, D$. Thus the particle and entropy densities are the same. While classically the collision time is zero for the densely packed liquid, quantum mechanically it is bounded from below by the Heisenberg uncertainty principle. Thus,

$$
\frac{\eta}{s} \geq \frac{\hbar}{4 \pi k_{B}}
$$

which is the same as the CFT limiting value. Turning the argument around through Eq. (50) implies an upper bound on the diffusion constant in a strongly coupled liquid, namely

$$
\frac{D}{\sigma_{0}} \leq \frac{k_{B} T}{12 h}
$$

in three dimensions, where the cross section $\sigma_{0}=8 \pi r_{0}^{2}$.

Let us now turn to cold atomic gases and repeat the same argument once more. It is simpler to imagine a Fermi gas in a vertical gravity field, for which we will rerun Einstein's derivation. We note that the trap field actually fulfills the same role. Using either Thomas-Fermi or hydrostatic calculations one finds that the Fermi momentum for non-relativistic quasiparticles in a gravitational field is

$$
\hbar k_{F}=\left(2 m^{*}(\mu-m g x)\right)^{1 / 2},
$$

where $m^{*}$ is the quasiparticle mass and $m$ is its bare gravitational mass. The normal Fermi density in a weak gravitational field is

$$
\frac{n(x)}{n(0)}=\left(1-\frac{m g x}{\mu}\right)^{3 / 2} \approx e^{-3 m g x /(2 \mu)}
$$

Using Eq. (55) and repeating steps which led to Eq. (51) one gets,

$$
\frac{\eta}{1 /\left(r_{0} \lambda^{2}\right)}=\frac{1}{3 \pi}(\mu \tau)
$$

with a diffusion constant

$$
D=\frac{\mu}{9 \pi r_{0} \eta}
$$

In the infinite coupling limit $\lambda$ becomes $r_{0}$ and the system is again closely packed. The Heisenberg uncertainty principle stipulates that the shortest collision time is dictated by the largest available quasiparticle energy, namely $\mu \tau \geq \hbar / 2$. Thus the new bound on the viscosity

$$
\frac{\eta}{\hbar n}=\alpha_{\eta} \geq \frac{1}{6 \pi} \text {. }
$$

Although Eq. (58) was derived for non-relativistic particles, its insensitivity to the quasiparticle velocities imply that it should hold in the relativistic case as well. Equation (58) implies an upper bound on the diffusion constant

$$
\frac{D}{\sigma_{0}} \leq \frac{\mu}{18 h},
$$

in trapped cold Fermions in three dimensions.

\section{CONCLUSIONS}

Strongly interacting systems, in relativistic field theories (QCD, CFL) and in condensed matter physics (strongly coupled QED plasma, ultracold atoms near the Feshbach resonance), are radically different from gas-like weakly interacting systems. The best way to see that is not via the equation of state and related thermodynamical observables, but with the help of transport properties.

As we have argued above, all such systems are nearideal liquids, and thus the natural tool one should use to describe those are standard viscous hydrodynamics. Indeed, one gets very good description of the "elliptic flow", the frequency and damping of the lowest collective oscillations.

Furthermore, the viscosity extracted from the data are shown to be very different from what is expected on the basis of binary collisions and in the weak coupling regime. We compared the minimal experimental value of the viscosity (or maximal rescattering rate) Eq. (27), with results of standard kinetic theory. Even without Pauli blocking and with maximal (unitary limited) binary cross section the kinetic theory fails to reproduce the data. We have also shown that the kinetic theory describes damping far from Feshbach resonance, where the the weakly coupled gas-like regime is valid. We conclude that the system of optically trapped ultracold atoms near the Feshbach resonance, like other strongly coupled systems discussed in section $\mathrm{V}$, is not a gas but rather a near-ideal liquid. Since oscillations are maximal at the surface, this small viscosity does not refer to superfluidity and is property of the normal component of the liquid making. Thus, the the properties of the normal component of the atomic system near the Feschbach resonance 
is quite different from that of liquid ${ }^{4} H e$ below the $\lambda$ point.

At infinite coupling the constituents are effectively large and densely packed. The packing fluctuates over short time scales dictated by the Heisenberg uncertainty principle. These physical insights together with Einstein's description of diffusion in viscous liquids, allow for a simple derivation of the viscosity to entropy density ratio established using CFT. We have extended this derivation to cold Fermi systems and derived an even lower bound for the viscosity to the particle density ratio.

It would be quite important to make more accurate measurements of the damping rate in order to see how close is the minimal viscosity to the theoretical bound. The temperature dependence of the damping rate would clarify the issue of Pauli blocking. We expect that the temperature dependence should be weak and not even close to what binary collision theory for Fermi gas predicts: the system is not even qualitatively close to a Fermi gas at the strong coupling limit.

Temperature dependence is also crucial for understanding of the transition to superfluidity which was avoided in this paper. We argued that in supercomponent resigns in the interior of the system, which is not very important for elliptic flow or oscillations we discussed. It seems that the lowest oscillation mode is well described by the one-component hydrodynamics: however it is of course quite likely that some higher excitations are analogous not to the usual sound but to other sounds known for superfluid liquid He.

One should also develop a theory of the strongly coupled systems beyond quite schematic mixtures of the BCS superconductor and ideal Bose gas of molecules. So far, such models can approximately reproduce the equation of state but not the transport properties. Numerical simulations of larger scales can also be helpful: perhaps one should complement the equation of state calculations by measurements of long-time correlators related by Kubo relations to transport coefficients.

\section{ACKNOWLEDGMENTS}

We thank Gerry Brown for numerous and very helpful discussions on Fermi liquids. We also thank H.T.C. Stoof for a useful discussion at the early stage of this work and R. Grimm. We especially grateful to J.E. Thomas for helpful correspondence. This work was partially supported by the US-DOE grants DE-FG02-88ER40388 and DE-FG03-97ER4014.

[1] H. Heiselberg, Phys. Rev. A63 (2001) 043606; T.-L. Ho,
Phys. Rev. Lett. 92 (2004) 090402; G.A. Baker, Jr., Phys. Rev. C60 (1999) 054311.

[2] J. Kinast et al., Phys. Rev. Lett. 92 (2004) 150402.

[3] J. Kinast et al., Phys. Rev. A70 (2004) 051401(R).

[4] M. Bartenstein et al., Phys. Rev. Lett. 92 (2004) 203201.

[5] E.V. Shuryak and I. Zahed, Phys. Rev. C70 (2004) 021902; for a review see E.V. Shuryak, hep-ph/0405066.

[6] K.M. O'Hara et al., Science 298 (2002) 2179.

[7] T. Bourdel et al., Phys. Rev. Lett. 91 (2003) 020402.

[8] M.E. Gehm et al., Phys. Rev. A68 (2003) 011401(R).

[9] L.D. Landau and E.M. Lifshitz, Fluid Mechanics, Pergamon, Oxford, 1959.

[10] C.J. Pethick and H. Smith, Bose-Einstein Condensation in Dilute Gases, Cambridge University Press, 2002.

[11] H. Heiselberg, Phys. Rev. Lett. 93, (2004) 040402.

[12] G.M. Kavoulakis, C.J. Pethick and H. Smith, Phys. Rev. A57 (1998) 2938.

[13] S. Gupta et al., Phys. Rev. Lett. 92 (2004) 100401.

[14] D. Teaney, J. Lauret and E.V. Shuryak, Phys. Rev. Lett. 86 (2001) 4783; for details see nucl-th/0110037.

[15] D. Teaney, J. Phys. G30 (2004) S1247.

[16] A. Nakamura and S. Sakai, Phys. Rev. Lett. 94 (2005) 072305.

[17] S. Ichimaru et al., Phys. Rep. 149C (1987) 91.

[18] J. Maldacena, Adv. Theor. Math. Phys. 2 (1998) 231; Phys. Rev. 80 (1998) 4859 and references therein.

[19] S.S. Gubser, I.R. Klebanov and A.A. Tseytlin, Nucl. Phys. B534 (1998) 202; C.P. Burgess, N.R. Constable, R.C. Myers, JHEP (1999) 9908; C. Kim and S.J. Rey, Nucl. Phys. B564 (2000) 430.

[20] G. Policastro, D.T. Son and A.O. Starinets, Phys. Rev. Lett. 87 (2001) 081601.

[21] A. Buchel, J.T. Liu and A.O. Starinets, hep-th/0406264.

[22] A.O. Starinets, Phys. Rev. D66 (2002) 124013.

[23] X. Xia and P.G. Wolynes, Phys. Rev. Lett. 86 (2001) 5526. 